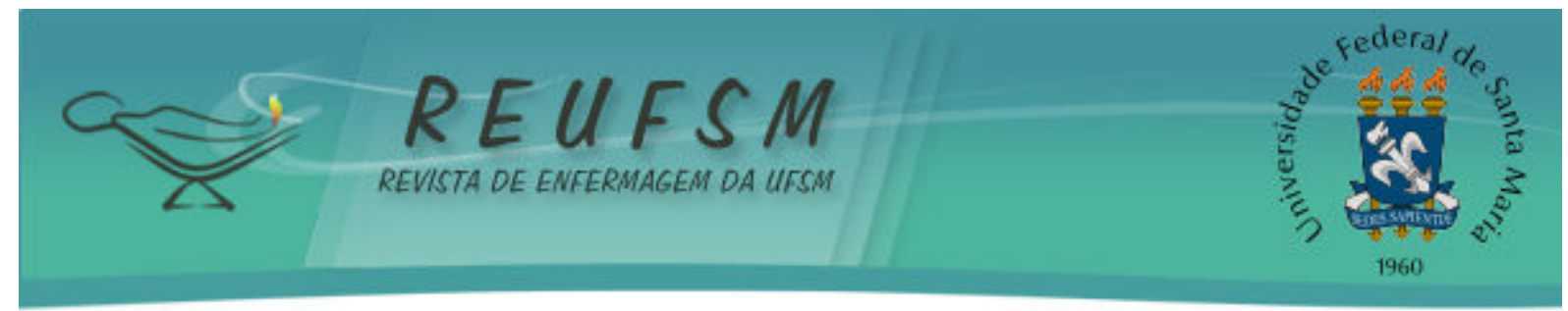

ARTIGO ORIGINAL

\title{
USO DE INTERVENÇÃO MUSICAL EM PACIENTES INTERNADOS EM UNIDADE DE TERAPIA INTENSIVA: ESTUDO PILOTO
}

\section{USE OF MUSICAL INTERVENTION IN PATIENTS IN THE INTENSIVE CARE UNIT: PILOT STUDY}

\section{USO DE INTERVENCIÓN MUSICAL EN PACIENTES INTERNADOS EN UNIDAD DE CUIDADOS INTENSIVOS: ESTUDIO PILOTO}

Lara Adrianne Garcia Paiano ${ }^{1}$ Luciana Magnani Fernandes ${ }^{2}$

\section{Doi: $10.5902 / 2179769213015$}

RESUMO: Objetivo: investigar as alterações geradas sobre os sinais vitais e nível de consciência com a aplicação da intervenção musical através da audição em pacientes sedados e não sedados, com trauma crânio encefálico, em uma unidade de terapia intensiva de um hospital de ensino. Método: estudo piloto quase-experimental, quantitativo. Participaram 10 pacientes, que receberam 20 sessões de intervenção musical. Para o tratamento dos dados foram utilizados os testes T-Student e ANOVA. As variáveis avaliadas foram: nível de consciência, frequência respiratória e cardíaca, pressão arterial e temperatura. Resultados: não houve alterações significativas nas variáveis aferidas. Observou-se através das médias, alterações dos parâmetros e no grupo de pacientes não sedados houve maior variabilidade das médias. Conclusão: a música pode ser utilizada como um estímulo a pacientes hospitalizados, podendo ser utilizado como recurso terapêutico e proporcionar um cuidado integral, individualizado e mais humanizado.

Descritores: Música; Enfermagem; Unidades de terapia intensiva.

ABSTRACT: Objective: to investigate the changes generated on vital signs and level of consciousness with the application of musical intervention through hearing in sedated and non-sedated patients with head trauma, in an intensive care unit of a teaching hospital. Method: quasi-experimental quantitative pilot study. 10 patients participated, having received 20 sessions of musical intervention. For the processing of the data, the tests T-Student and ANOVA were used. The variables evaluated were: level of consciousness, respiratory and heart rate, blood pressure and temperature. Results: there were no significant changes in the variables measured. Parameter changes were observed through the averages. In the group of non-sedated patients, there was higher variability of the average. Conclusion: music can be used as a stimulus for hospitalized patients. It could be used as a therapeutic resource and provide a comprehensive, individualized and more humanized care.

Descriptors: Music; Nursing; Intensive care units.

RESUMEN: Objetivo: investigar los cambios provocados en los signos vitales y en el nivel de conciencia durante la aplicación de intervención musical mediante audición en pacientes sedados y no sedados, con traumatismo craneoencefálico, de una unidad de

\footnotetext{
${ }^{1}$ Enfermeira Especialista em Gerenciamento de Enfermagem em Clínica Médica e Cirúrgica, Docente do curso de Enfermagem da Universidade Estadual do Oeste do Paraná - UNIOESTE, Campus Cascavel, PR, Brasil. Email: laraagp@hotmail.com.br.

${ }^{2}$ Enfermeira Doutora em Enfermagem, Docente do curso de Enfermagem da Universidade Estadual do Oeste do Paraná - UNIOESTE, Campus Cascavel, PR, Brasil. Email: lumagna@terra.com.br.
} 


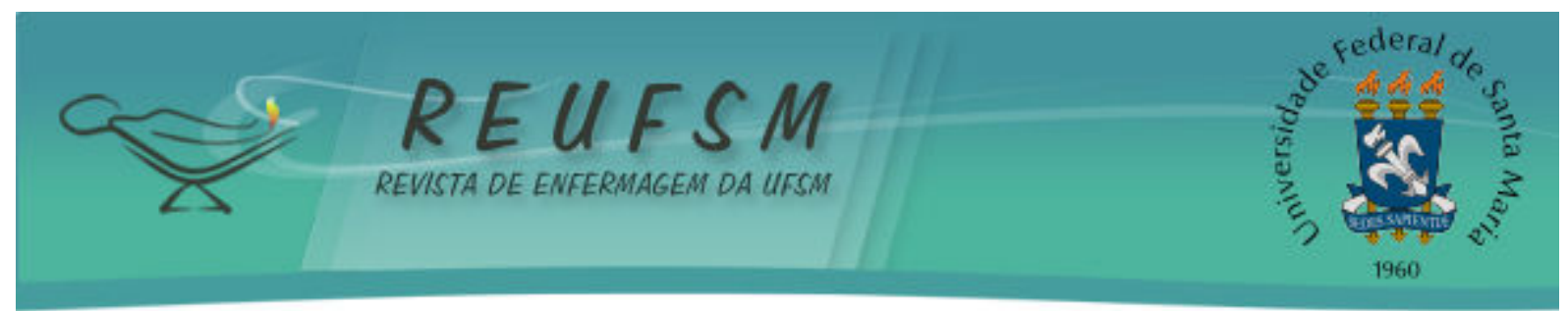

cuidados intensivos de un hospital universitario. Método: estudio piloto cuasiexperimental, cuantitativo. Participaron 10 pacientes, que recibieron 20 sesiones de intervención musical. Para tratamiento de los datos, se utilizaron las pruebas T-Student y ANOVA. Las variables evaluadas fueron: nivel de conciencia, frecuencia respiratoria y cardíaca, presión arterial y temperatura. Resultados: no hubo cambios significativos en en las variables medidas. Se observó a través de las medias cambios en los parámetros, y en los pacientes no sedados hubo mayor variabilidad de promedios. Conclusión: la música se puede utilizar como estímulo a los pacientes hospitalizados, pudiendo ser utilizada como un recurso terapéutico y proporcionar atención integral, individualizada y más humanizada.

Descriptores: Música; Enfermería; Unidades de cuidados intensivos.

\section{INTRODUÇÃO}

A música é tão antiga quanto a história da humanidade e evoluiu dentre os povos de acordo com seus costumes e tradições. Tem um significado importante na comunicação, sendo uma forma de linguagem transmitida pelas gerações e um meio de educar e ajudar a construir a personalidade do indivíduo. ${ }^{1}$

A música é considerada uma experiência universal, na qual, tudo pode ser compartilhado. Portanto, ela não deve ser tida apenas como um divertimento e como um meio de se educar e integrar o indivíduo à sociedade, mas, pode ainda ser utilizada no contexto clínico ${ }^{2}$, podendo auxiliar no cuidado em saúde.

No século XIX, a música foi muito utilizada e difundida em hospitais psiquiátricos e no tratamento de doenças psicossomáticas. Na enfermagem, o uso da música com finalidade terapêutica esteve presente desde os tempos de Florence Nightingale, em 1859, e anos depois, nas duas Grandes Guerras Mundiais na assistência aos soldados feridos em combates, para aliviar a dor física e emocional. ${ }^{3}$

Atualmente, profissionais da saúde, têm discutido as variadas manifestações e expressões trazidas pela música e suas influências em pacientes com diversas patologias e, com isso, estão introduzindo na assistência, a musicoterapia com intuito de oferecer um cuidado humanizado e promover a saúde do trabalhador. ${ }^{3}$

Estudos mostram os efeitos da música, comprovando que ela pode agir diretamente no corpo humano, diminuindo a pressão arterial, apresentar alterações na frequência cardíaca, alterações respiratórias e pode agir sobre o desconforto e auxiliar na tolerância da dor, fazer rir, chorar, de modo consciente e não consciente. ${ }^{1-4}$

Foram encontrados dados na literatura que apresentaram os benefícios da música para melhorar a resposta e coordenação motora em casos de paralisia cerebral e distrofias musculares, em pacientes com doenças degenerativas e traumas cerebrais, pacientes com deficiências mentais e sofrimento psíquico, no tratamento de disfunções da linguagem e da fala, no tratamento de dores agudas e crônicas, para controle da respiração e redução de tensão emocional, em casos de doenças terminais, no pré e pós-operatório, entre outros. ${ }^{2}$

A música pode ser percebida pelas mesmas áreas do cérebro que recebem informações correspondentes às emoções e sensações, sendo influente nos sentimentos e consequentemente no comportamento dos indivíduos, além de contribuir com o desenvolvimento do raciocínio lógico, memória, capacidade de comunicação e compreensão da linguagem. Pode aumentar o bom humor, induzir à calma e o sono, o relaxamento muscular, beneficiando o sistema nervoso central. ${ }^{1}$

0 uso da música quando aplicada em sua forma terapêutica diminui os níveis de estresse, ansiedade e desconfortos, principalmente em ambiente hospitalar, apresentando- 


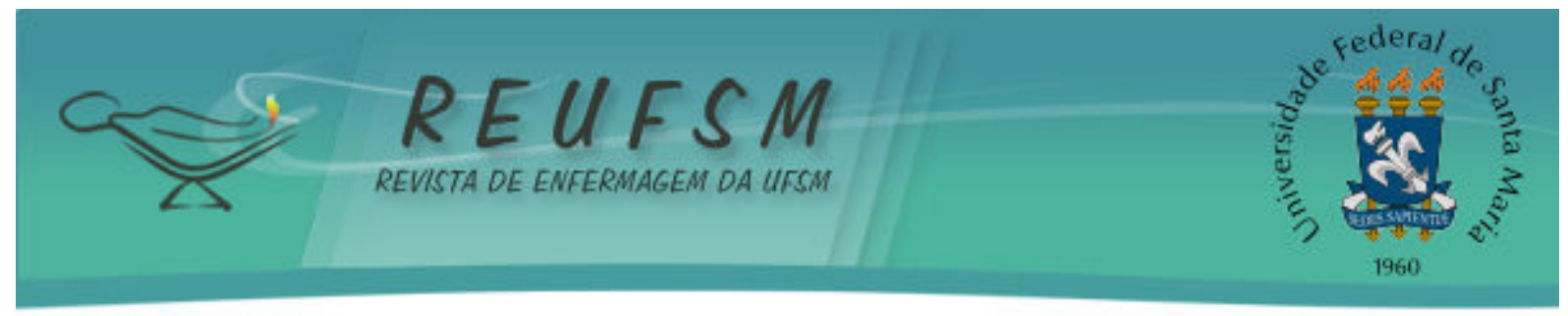

se eficaz na assistência de enfermagem, proporcionando um cuidado humanizado e promovendo interação entre paciente e equipe e entre equipe multidisciplinar. ${ }^{3}$

Assim, partindo do pressuposto de que a música tem influência no comportamento humano e que ela pode ser utilizada como uma terapia complementar na área assistencial, a intenção em desenvolver este estudo piloto teve como questão norteadora o interesse em investigar se a música pode gerar alterações sobre os sinais vitais e sobre o nível de consciência de pacientes hospitalizados. Portanto, o estudo teve como objetivo investigar as alterações geradas sobre os sinais vitais e nível de consciência com a aplicação de intervenção musical em pacientes sedados e não sedados, com trauma crânio encefálico, em uma unidade de terapia intensiva de um hospital de ensino.

\section{MÉTODO}

Estudo piloto quase-experimental, quantitativo, realizado com pacientes vítimas de Trauma Crânio Encefálico (TCE), internados na Unidade de Terapia Intensiva para adultos de um hospital de ensino do Estado do Paraná, no período entre agosto e novembro de 2013.

Os pacientes foram selecionados por meio de investigação diária ao prontuário eletrônico e foram incluídos aqueles que atendiam os seguintes critérios: diagnóstico confirmado de TCE; sem sedação com escore da Escala de Coma de Glasgow (ECG) entre três e oito pontos; sedados avaliados por meio da Escala de Sedação de Ramsay (ESR) com escores entre um e seis; submetidos à ventilação mecânica invasiva ou não invasiva; com estabilidade hemodinâmica; com função auditiva preservada e com autorização da família ou responsável para a participação do paciente no estudo. Foram excluídos os pacientes com suspeita e em investigação para confirmação diagnóstica de morte encefálica.

Após entrevista com familiar ou responsável e autorização para participação do paciente no estudo foi realizada, diariamente, a aplicação da intervenção musical proposta, a qual consistia na instalação de fones de ouvido descartáveis nos pacientes para audição de músicas pré-selecionadas pelo pesquisador. Três músicas foram selecionadas e armazenadas em um aparelho de mídia player portátil, totalizando 20 minutos de audição musical para cada sessão de aplicação da intervenção. As músicas selecionadas eram peças eruditas, sendo duas de Wolfgang Amadeus Mozart e uma de Heitor Villa-Lobos.

A primeira música selecionada foi o Primeiro Movimento da Sonata para dois Pianos em Ré maior K448 de Mozart (9') que tem sido bastante citada na literatura com o fenômeno chamado "Efeito Mozart". Este fenômeno consiste em efeitos gerados com a audição da referida peça, sendo eles, a melhora no desempenho neuropsicológico em provas espaciais e alterações neurofisiológicas em estudantes universitários evidenciadas por pesquisadores. ${ }^{7}$ A segunda música selecionada foi o Primeiro Movimento do Concerto para violino $\mathrm{n}^{\circ} 4$ em Ré Maior k218 (7’52”) de Mozart, que também se destaca entre as obras do compositor.

A terceira música selecionada foi a Toccata Trenzinho Caipira da peça Bachianas Brasileiras n.4 de Heitor Villa-Lobos (4'30"), obra erudita brasileira que descreve o movimento de uma locomotiva através dos sons dos instrumentos da orquestra, apresentando frases melódicas e rítmicas que propiciam a impressão e percepção do ouvinte, de movimento constante. ${ }^{8}$

Os dados foram registrados em um instrumento próprio, considerando-se as seguintes variáveis: idade; sexo; diagnóstico; tempo de internação na UTI; tipo de suporte respiratório; modos e parâmetros ventilatórios; nível de consciência; sinais vitais; tipo de sedação e observações/informações pertinentes. 


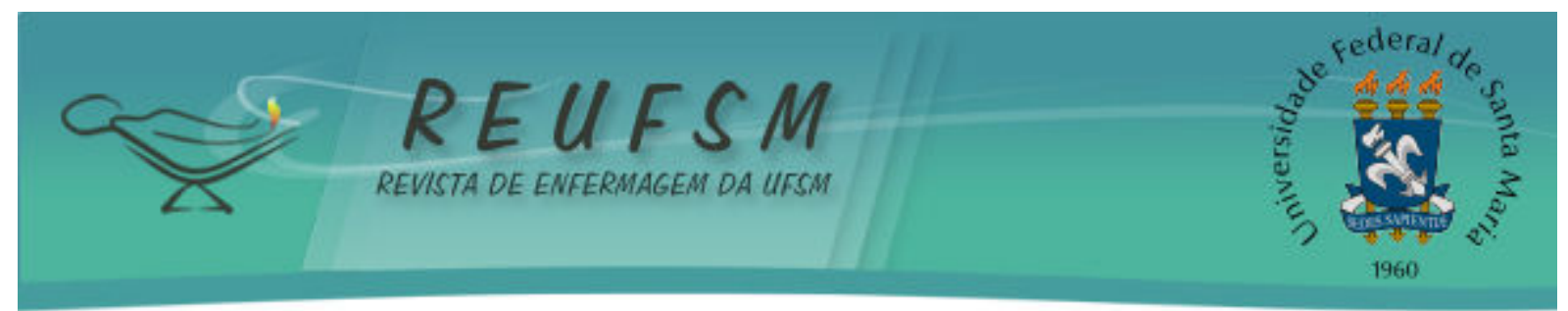

Participaram do estudo 10 pacientes, com os quais foram realizadas 20 sessões de intervenção musical. Dez sessões foram realizadas com pacientes sedados e dez com pacientes não sedados.

No que se refere aos pacientes sedados, três pacientes receberam duas sessões e um paciente foi submetido a quatro sessões. Quanto aos pacientes não sedados, quatro pacientes receberam uma sessão e um paciente recebeu duas sessões. No entanto, um dos participantes foi submetido a duas sessões quando estava sedado e a duas sessões quando não estava sedado.

Os sinais vitais foram registrados em três momentos: antes da intervenção musical, durante a intervenção (10 minutos após o início) e cinco minutos após o término da intervenção (25 minutos após a primeira coleta). Os dados foram coletados a partir da aferição manual ou da verificação através de monitores multiparamétricos.

Durante as sessões os pacientes recebiam somente a intervenção musical, não era realizado nenhum procedimento pela equipe de profissionais da UTI. Para avaliar o nível de consciência dos pacientes sedados foi utilizada a ESR e para os pacientes não sedados a ECG.

O TCE foi classificado em grave, moderado e leve conforme a pontuação na ECG, sendo considerados graves pacientes que pontuam entre três e oito pontos, moderados pacientes que pontuam entre oito e 12 pontos e leve pacientes que pontuam de 13 a 15 pontos. $^{9}$

As variáveis analisadas foram: nível de consciência (nc), frequência respiratória (fr), frequência cardíaca (fc), pressão arterial sistólica (pas), pressão arterial diastólica (pad) e temperatura corporal (t). Para cada variável foram registradas três medidas, antes, durante e após a intervenção musical e para identificá-las, junto às siglas das variáveis, os números, 1 , 2 e 3 foram acrescentados a fim de representar os momentos das três medidas (ex: fr1: frequência respiratória antes do início da música; fr2: frequência respiratória durante a música e; fr3: frequência respiratória após 05 minutos do término da música).

Os dados foram sistematizados e analisados utilizando-se metodologia estatística, com apresentação de valores médios, desvio padrão e percentuais. Para verificar se ocorreram alterações nos sinais vitais durante as sessões de música, utilizou-se um modelo em blocos casualizados dado por $Y_{i j k}=\mu+T_{i}+B_{j}+\varepsilon_{i j k}$ onde $\mu$ é um efeito médio geral, $T_{i}$ corresponde ao efeito do $i$-ésimo tratamento, $B_{j}$ o efeito do $j$-ésimo bloco e $\varepsilon_{\mathrm{ijk}}$ um efeito aleatório, supostamente com distribuição normal de média zero e variância constante $\varepsilon$, todos associados a variável resposta (sinal vital aferido) $Y_{i j k}$. Para o teste da hipótese de ausência de significância dos efeitos foi empregado uma análise de variância (ANOVA) com $5 \%$ de significância, e para as comparações das médias dos efeitos estudados sobre as variáveis resposta foi empregado o teste t-Student, também com $5 \%$ de significância. Todas as análises foram feitas empregando-se o programa estatístico R. ${ }^{10}$

O estudo foi submetido à apreciação do Comitê de Ética em Pesquisa da universidade e aprovado pelo Parecer 031/2013 em atendimento ao previsto na Resolução 466/2012 do Conselho Nacional de Saúde. ${ }^{11}$

\section{RESULTADOS E DISCUSSÕES}

As intervenções musicais foram realizadas em 10 pacientes, sendo sete (70\%) do sexo masculino e três (30\%) do sexo feminino. Em relação à faixa etária destes pacientes, a média de idade foi de 34,3 anos ( $\mathrm{DP} \pm 17,2 / \mathrm{md} \mathrm{29,5)}$. Três $(30 \%)$ tinham idade entre $17 \mathrm{e}$ 25 anos, dois (20\%) entre 26 e 30 anos, dois (20\%) entre 31 e 35 anos, um (10\%) entre 36 e 40 anos, um (10\%) entre 56 e 60 anos e um (10\%) entre 66 e 70 anos (Quadro 1). 


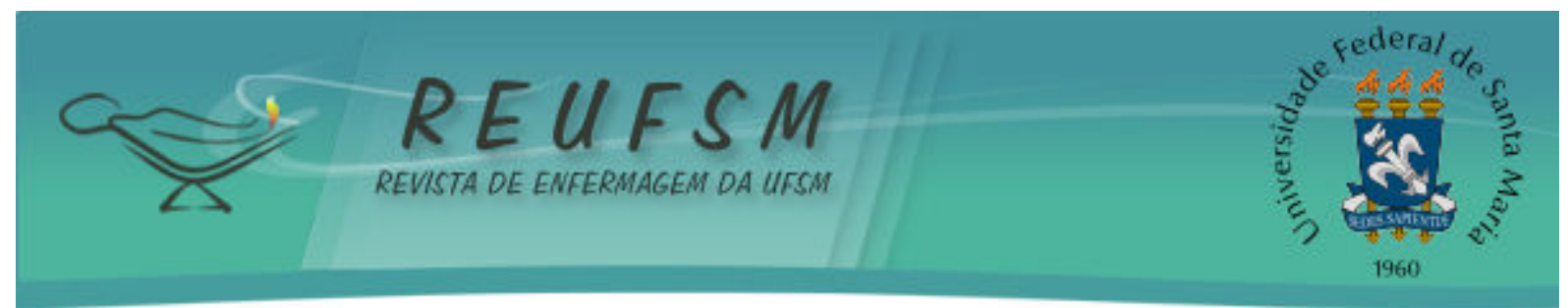

Todos os pacientes eram vítimas de TCE. Segundo o Ministério da Saúde a ocorrência do TCE se dá predominantemente no sexo masculino e acomete principalmente os adultos jovens como evidenciado nos dados acima. Possui altas taxas de mortalidade e produz grande impacto social e na qualidade de vida do indivíduo devido às sequelas. Geralmente necessitam de internação em unidades de terapia intensiva devido ao comprometimento crânio-encefálico e complicações sistêmicas. ${ }^{9}$

O TCE consiste na ocorrência de lesões das estruturas anatômicas cerebrais decorrentes de traumas externos. Pode causar diminuição ou alteração de consciência, resultando em distúrbios no funcionamento físico, cognitivo, comportamental ou emocional. $^{9}$

Em relação à classificação do TCE, sete pacientes que receberam a intervenção (70\%) foram diagnosticados como grave e, três $(30 \%)$ como moderado. Quanto ao comprometimento do tecido cerebral e lesões causadas com o traumatismo, três pacientes apresentaram fraturas de osso temporal, dois apresentaram fraturas de osso occipital, dois apresentaram hematoma subdural, um apresentou hematoma extradural, cinco apresentaram hemorragia subaracnóidea, dois apresentaram edema cerebral e um não apresentou alterações. Estes dados foram coletados a partir dos laudos de exame de tomografia computadorizada e através dos relatórios médicos.

Dependendo da extensão e gravidade da área acometida pela lesão, as sequelas físicas, cognitivas e emocionais que podem ser desencadeadas são: comprometimento sensório - motor (perda da capacidade tátil, temperatura, dor, textura e propriocepção), alterações músculo-esqueléticas (diminuição no tônus muscular, espasticidade, contraturas e evolução para deformidades), hemiplegia e hemiparesia, distúrbios cardiovasculares, endócrinos e respiratórios, diminuição da capacidade de aprendizagem, de concentração, da memória, disfunções linguísticas, depressão, ansiedade, apatia e distúrbios do sono, entre outros. ${ }^{9}$

Quanto às causas do TCE: um paciente $(10 \%)$ foi vítima de atropelamento, uma (10\%) vítima de queda de altura, duas $(20 \%)$ vítimas de espancamento, duas (20\%) vítimas de capotamento com ejeção do veículo, três $(30 \%)$ vítimas de acidentes automobilísticos com colisão entre automóvel e motocicleta e uma (10\%) vítima de acidente automobilístico com colisão entre automóvel e automóvel.

O Ministério da Saúde aponta que $50 \%$ dos casos de TCE ocorrem devido acidentes de trânsito, seguidos de quedas (30\%), principalmente com idosos, e devido a causas violentas (20\%), como verificado nos dados acima e apresentado no Quadro $1 .^{9}$

Quanto ao período de internação na unidade de terapia intensiva, o tempo médio de internação dos pacientes foi de 14 dias, variando entre cinco e 22 dias ( $D P \pm 5,24$ dias). 


$\begin{array}{cccccccc}\text { Faciente } & \text { Sexo } & \text { Idade } & \text { TCE } & \text { Lesão } & \text { Causa } & \text { Dias } \\ \text { UTI }\end{array}$

Quadro 1 - Pacientes com TCE internados em uma unidade de terapia intensiva.

\section{Nível de consciência e sinais vitais}

Embora estudos já venham apresentando diversas manifestações e efeitos fisiológicos produzidos no organismo humano através de intervenções utilizando a música, comprovando que pode haver alterações nos sinais vitais, nos níveis de dor e de ansiedade, na aceleração do metabolismo, nos estados de humor, nas respostas cognitivas, de memória e aprendizagem, os resultados encontrados neste estudo não mostraram alterações estatisticamente significativas. 0 teste T-Student e o teste ANOVA indicaram a não significância ( $p$-valor $>0,05$ ) para as variáveis (sinais vitais aferidos) sugerindo a ausência do efeito da música nos valores obtidos.

Alguns dos pacientes receberam mais que uma intervenção, em momentos (dias) diferentes. Portanto, esta análise, é baseada nas sessões de música realizadas, ou seja, 10 sessões foram realizadas com os pacientes sedados e 10 sessões com os pacientes sem sedação. Em relação ao nível de consciência, nas 10 sessões com os pacientes sedados, todos, pontuaram seis pontos na ESR nas três avaliações realizadas, ou seja, antes, durante e após a intervenção (Quadro 2). A sedação com infusão contínua de fármacos depressores do sistema nervoso central é indicada para a manutenção e estabilização hemodinâmica, metabólica e respiratória e para redução da ansiedade e agitação de pacientes críticos ${ }^{12}$, condições estas, apresentadas pelos pacientes com TCE, sedados, que participaram do estudo. 


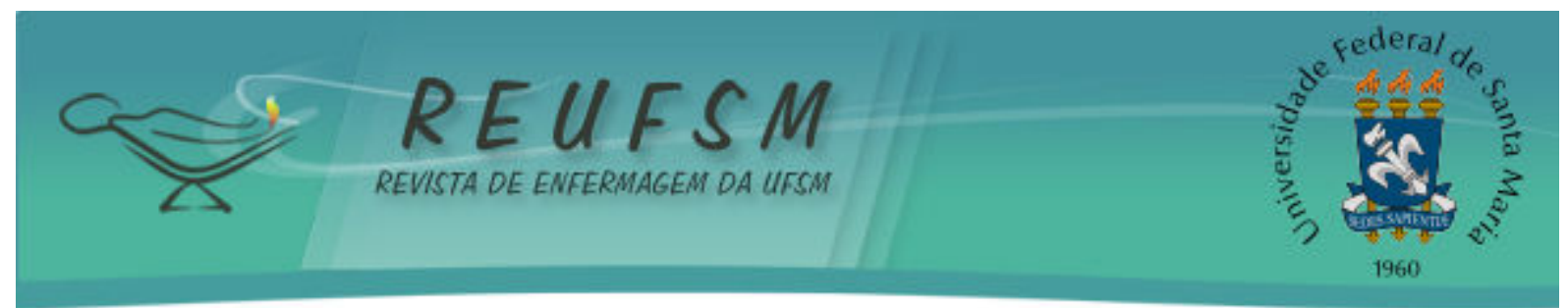

Os pacientes não sedados apresentaram quadros neurológicos semelhantes, com a média de escore da ECG de 4,2 pontos $(D P \pm 1,22)$, com variação entre quatro e seis. Os pacientes não apresentaram alterações do nível de consciência durante as sessões de música (Quadro 2). Estes pacientes já não estavam com uso de sedativos, mantinham suporte ventilatório com a ventilação mecânica e alguns apresentavam alguma resposta só ao estímulo doloroso.

\begin{tabular}{|cccc}
\hline $\begin{array}{c}\text { Sessão com } \\
\text { pacientes sedados }\end{array}$ & $\begin{array}{c}\text { Pontuação } \\
\text { na ESR }\end{array}$ & $\begin{array}{c}\text { Sessão com } \\
\text { pacientes não } \\
\text { sedados }\end{array}$ & $\begin{array}{c}\text { Pontuação } \\
\text { na ECG }\end{array}$ \\
01 & 06 & 01 & 06 \\
02 & 06 & 02 & 06 \\
03 & 06 & 03 & 03 \\
04 & 06 & 04 & 03 \\
05 & 06 & 05 & 04 \\
06 & 06 & 06 & 04 \\
07 & 06 & 07 & 03 \\
08 & 06 & 08 & 03 \\
09 & 06 & 09 & 05 \\
10 & 06 & 10 & 05 \\
Média & 06 & Média & 4,2 \\
Desvio-padrão & 0 & Desvio-padrão & $\pm 1,22$ \\
\hline Quadro 2 - Pontuação nas escalas de avaliação do nível de consciência de pacientes sedados e não
\end{tabular}

Embora os resultados encontrados não tenham apresentado alterações estatisticamente significativas, quanto aos sinais vitais, quando analisadas as medidas das variáveis e quando comparados os resultados das sessões em pacientes sedados e não sedados, foram observadas variações através das médias e desvio-padrão, dados estes, descritos no Quadro 3. 


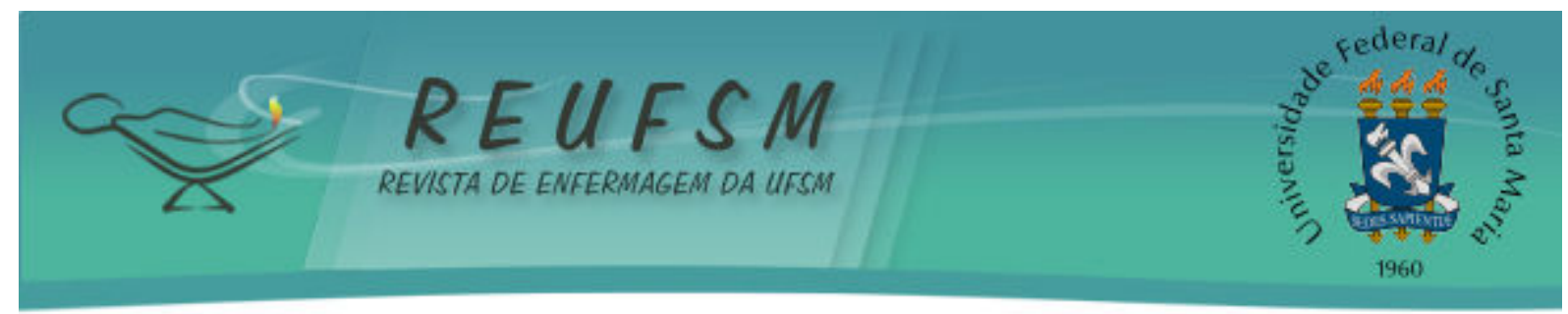

\begin{tabular}{|c|c|c|c|c|c|c|c|}
\hline \multicolumn{8}{|c|}{ Pacientes sedados ( $n=10$ sessões) Pacientes não sedados ( $n=10$ sessões) } \\
\hline FR & $M$ & DP & p-valor* & FR & $M$ & DP & p-valor* \\
\hline Fr1 & 22,1 & 2,2 & Fr1Fr2 1,0000 & Fr1 & 20,9 & 4,8 & $\begin{array}{l}\text { Fr1Fr2 } \\
0,7421\end{array}$ \\
\hline Fr2 & 22,1 & 2,2 & Fr1Fr3 1,0000 & Fr2 & 21,8 & 6,9 & $\begin{array}{l}\text { Fr1Fr3 } \\
0,8678\end{array}$ \\
\hline Fr3 & 22,1 & 2,2 & Fr2Fr3 1,0000 & Fr3 & 21,3 & 6,7 & $\begin{array}{l}\text { Fr2Fr3 } \\
0,8623\end{array}$ \\
\hline FC & M & DP & p-valor* & FC & M & DP & p-valor* \\
\hline Fc1 & 88,9 & 10,5 & $\begin{array}{l}\text { Fc1Fc2 } \\
0,9836\end{array}$ & Fc1 & 94,4 & 17,6 & $\begin{array}{l}\text { Fc1Fc2 } \\
0,7942\end{array}$ \\
\hline Fc2 & 88,8 & 10,8 & $\begin{array}{l}\text { Fc1Fc3 } \\
0,9677\end{array}$ & Fc2 & 96,6 & 19,4 & $\begin{array}{l}\text { Fc1Fc3 } \\
0,3554\end{array}$ \\
\hline Fc3 & 88,7 & 11,2 & $\begin{array}{l}\text { Fc2Fc3 } \\
0,9841\end{array}$ & Fc3 & 102,3 & 19,5 & $\begin{array}{l}\text { Fc2Fc3 } \\
0,5216\end{array}$ \\
\hline PAS/PAD & M & DP & p-valor* & PAS/PAD & M & DP & p-valor* \\
\hline Pas1 & 129 & 17,2 & $\begin{array}{c}\text { Pas1Pas2 } \\
0,7888\end{array}$ & Pas1 & 144,8 & 27 & $\begin{array}{c}\text { Pas1Pas2 } \\
0,2994\end{array}$ \\
\hline Pas2 & 126,9 & 17,3 & $\begin{array}{c}\text { Pas1Pas3 } \\
0,6459\end{array}$ & Pas2 & 158,8 & 31,3 & $\begin{array}{c}\text { Pas1Pas3 } \\
0,3121\end{array}$ \\
\hline Pas3 & 125,2 & 19 & $\begin{array}{c}\text { Pas2Pas3 } \\
0,8351\end{array}$ & Pas3 & 157,7 & 28,4 & $\begin{array}{c}\text { Pas2Pas3 } \\
0,9354\end{array}$ \\
\hline Pad1 & 73,5 & 9,2 & $\begin{array}{c}\text { Pad1Pad2 } \\
0,685\end{array}$ & Pad1 & 78,5 & 8,9 & $\begin{array}{c}\text { Pad1Pad2 } \\
0,6193\end{array}$ \\
\hline Pad2 & 71,6 & 11,2 & $\begin{array}{c}\text { Pad1Pad3 } \\
0,4524\end{array}$ & Pad2 & 80,6 & 9,6 & $\begin{array}{c}\text { Pad1Pad3 } \\
0,7537\end{array}$ \\
\hline Pad3 & 69,7 & 12,5 & $\begin{array}{c}\text { Pad2Pad3 } \\
0,7262\end{array}$ & Pad3 & 77,2 & 9,2 & $\begin{array}{c}\text { Pad2Pad3 } \\
0,432\end{array}$ \\
\hline $\mathbf{T}$ & $M$ & DP & p-valor* & $\mathrm{T}$ & $M$ & DP & p-valor* \\
\hline $\mathrm{T} 1$ & 36,8 & 0,5 & T1T2 0,9648 & $\mathrm{T} 1$ & 37,1 & 0,4 & $\begin{array}{c}\text { T1T2 } \\
0,8748\end{array}$ \\
\hline $\mathrm{T} 2$ & 36,7 & 0,4 & T1T3 0,8882 & $\mathrm{T} 2$ & 37,1 & 0,4 & $\begin{array}{c}\text { T1T3 } \\
0,9167\end{array}$ \\
\hline T3 & 36,8 & 0,4 & T1T2 0,8469 & T3 & 37,1 & 0,4 & $\begin{array}{c}\text { T1T2 } \\
0,9581\end{array}$ \\
\hline
\end{tabular}

Quadro 3 - Comparação dos sinais vitais antes (Fr1; Fc1; Pas1; Pad1; T1), durante (Fr2; Fc2; Pas2; Pad2; T2) e após cinco minutos (Fr3; Fc3; Pas3; Pad3; T3), do término da intervenção musical nas sessões com pacientes sedados e não sedados.

Nota: *p-valor resultante da aplicação do teste T-Student para as comparações das médias em cada grupo de pacientes.

Nota: $M$ refere-se à média; DP refere-se a desvio-padrão.

A maioria dos pacientes com TCE classificados como grave e moderado, como a população deste estudo, evoluem com insuficiência respiratória necessitando de suporte ventilatório artificial, que está relacionado com o controle das pressões parciais dos gases sanguíneos, além de contribuir para o controle da pressão intracraniana, que na maioria dos casos, pode estar aumentada. ${ }^{13}$

Nas sessões de música realizadas com pacientes sedados, $100 \%$ estavam intubados com tubos orotraqueais e estavam submetidos ao modo ventilatório assistido-controlado. 


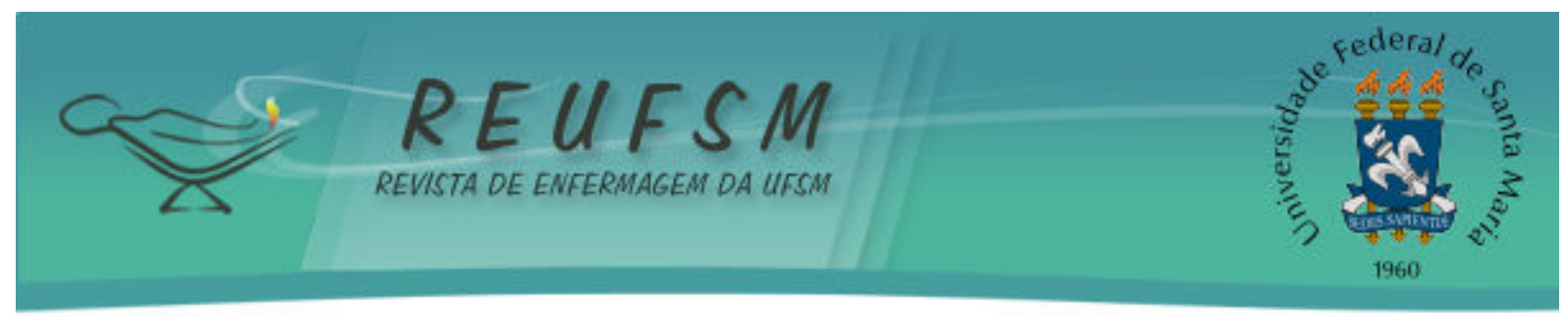

Ao analisarmos os valores das médias da frequência respiratória nestes pacientes, observou-se que os valores são iguais, não houve alterações antes, durante e após cinco minutos do término da intervenção musical.

0 modo assistido-controlado, ao qual os pacientes foram submetidos, consistia no disparo dos ciclos ventilatórios de acordo com a pressão negativa ou fluxo positivo realizado pelo paciente, sendo que a frequência respiratória pode variar de acordo com o disparo decorrente do esforço inspiratório do indivíduo, mantendo parâmetros de volume e fluxo corrente fixos e, caso o paciente não atinja um valor pré-determinado de sensibilidade para disparar o ventilador mecânico, o mesmo manterá um ciclo de frequências mínimas pré-fixadas. ${ }^{14}$

No grupo das sessões com pacientes não sedados, dois pacientes estavam submetidos ao modo ventilatório assistido-controlado e oito submetidos ao modo de ventilação mandatória intermitente sincronizada (SIMV), que é o modo no qual a frequência respiratória, o volume e fluxo inspiratório são programados, mas no qual o ventilador permite que haja ciclos espontâneos em sincronia com os disparos dos ciclos mandatórios. Pôde-se observar valores de frequência respiratória diferentes do préestabelecido. As alterações observadas nas médias das frequências respiratórias dos pacientes não sedados podem ser justificadas pelo uso do modo de ventilação SIMV, resultando dados de frequência respiratória de valores diferentes.

Quanto à frequência cardíaca e à pressão arterial sistólica observou-se que nas sessões com pacientes sedados houve uma discreta diminuição nos valores, enquanto que nas sessões com pacientes não sedados houve um aumento gradativo nos valores das médias, durante e após o término da intervenção musical. Além disso, os valores das médias comparando um grupo e outro mostraram que os valores dessas nos pacientes não sedados eram maiores que os valores das médias dos pacientes sedados.

Estudos apontam que quando se deseja proporcionar sensações de conforto e tranquilidade, as músicas mais indicadas são as que possuem ritmos mais lentos, melodias com tons mais graves e intensidade mais suave que promovem o relaxamento, redução do nível de ansiedade, da frequência respiratória e frequência cardíaca. Quando se deseja ampliar o estado de alerta, as músicas mais indicadas são as mais rápidas e com tons mais agudos, estas também podem ocasionar aumento da pressão arterial e das frequências respiratória e cardíaca. ${ }^{15,16}$

Nesse sentido, as músicas selecionadas possuem em sua estrutura constantes variações nas linhas melódicas, rítmicas, harmônicas, na intensidade e no andamento, componentes estes que influenciam diretamente nos efeitos gerados nos indivíduos. Alguns autores afirmam que devemos considerar a preferência musical do paciente para a intervenção, alertando sobre os benefícios e a possibilidade da ocorrência de desconfortos devido às antipatias com estilos de música e gosto individual ${ }^{6}$, porém, a escolha das três obras justificou-se por tratarem-se de obras importantes, por constituírem composições de alto teor artístico e no caso da primeira, por já ter sido empregada em outros estudos científicos. ${ }^{17}$

Quanto à temperatura corporal as médias observadas nos dois grupos de pacientes praticamente não apresentaram alterações e não houve alterações significativas entre as medidas. Resultados semelhantes foram encontrados em outro estudo, o qual também evidenciou que as alterações apresentadas não eram estatisticamente significantes na temperatura corporal. ${ }^{6}$

Além disso, é válido mencionar que os pacientes que participaram do estudo tinham em seus prontuários, na prescrição diária, a administração de antitérmicos e antimicrobianos devido a quadros de picos febris e infecções.

Estes resultados podem ter sido influenciados pelo processo de hospitalização e o estresse causado em pacientes internados em UTI, submetidos à ventilação mecânica, os 


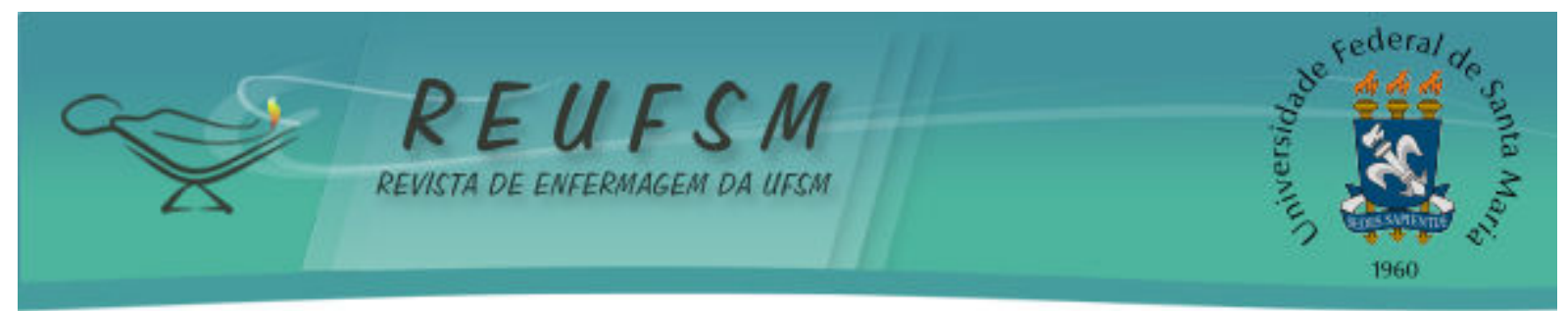

quais são mais intensos e evidentes devido a fatores como o período prolongado de internamento, efeitos colaterais da analgesia e da sedação, nível de consciência, infusão de medicamentos, imobilidade, frequência da aspiração orotraqueal, aumento dos níveis de ansiedade, evidenciados principalmente nas tentativas de desmame da ventilação mecânica, sensação de falta de ar, impossibilidade de falar, agitação, e o próprio histórico clínico do paciente, dentre outros.

As terapias alternativas, dentre elas a música, estão sendo difundidas cada vez mais, embora muitos profissionais de saúde ainda possuam receios e muitas dúvidas quanto à eficácia da música. Em se tratando do ambiente hospitalar e especificamente a UTI, muitas vezes vista como um ambiente frio, hostil e assustador, a aplicação da intervenção musical pode proporcionar momentos de relaxamento ou estímulos cognitivos, sensórios e motores e atuar como recurso facilitador na comunicação entre paciente, equipe multiprofissional e familiares, propiciando um cuidado integral, individualizado e mais humanizado.

A maior limitação deste estudo refere-se ao número reduzido de pacientes que atendiam aos critérios de inclusão no período destinado para a coleta de dados, o que influenciou diretamente na análise dos dados e nos resultados obtidos. No entanto, sendo este um estudo piloto, propõe-se a reavaliação das estratégias metodológicas para estudo futuro.

\section{CONSIDERAÇÕES FINAIS}

Os resultados apresentados mostraram que não houve alterações significativas no nível de consciência e nos valores dos sinais vitais aferidos nos dois grupos de pacientes (sedados e não sedados), mas, foram observados o aumento e a diminuição de alguns parâmetros e, que principalmente no grupo de pacientes não sedados houve maior variabilidade nos valores das médias. Evidenciamos que a música não gerou efeitos expressivos sobre as dados vitais ou no quadro clínico dos pacientes, nos dois grupos em estudo.

Vale ressaltar a necessidade de se desenvolver mais pesquisas e estudos que tragam discussões e produções científicas sobre a inserção de terapias alternativas na assistência de enfermagem e no cuidado ao paciente criticamente enfermo a fim de aprofundar o conhecimento já explorado e apresentar novas possibilidades terapêuticas.

A música pode ser utilizada como um estímulo à pacientes hospitalizados, considerando seu quadro clínico, servindo como recurso terapêutico. 0 enfermeiro, desde que detenha conhecimento, pode ser um dos profissionais facilitadores no processo de implantação, execução e avaliação da eficácia deste tipo de terapia, proporcionando uma assistência mais humanizada, buscando ampliar as formas e os meios de se realizar o cuidado.

\section{REFERÊNCIAS}

1. Simões MGN. Avaliação dos efeitos combinados do exercício físico e da música na motivação para o exercício, nos estados de humor e na função cognitiva. [dissertação] Gambelas: Faculdade de Ciências Humanas e Sociais da Universidade do Algarve, 2010. 173p.

2. Bréscia VP. A música como recurso terapêutico. In: Anais do XIV Encontro Paranaense, IX Congresso Brasileiro de Psicoterapias Corporais; 2009. Curitiba: Centro Reichiano, 2009. Disponível

em <http://www.centroreichiano.com.br/artigos/Anais\%202009/BRESCIA,\%20Vera\%20Pessagno \%20-\%20A\%20musica\%20como\%20recurso.pdf>Acesso em 20/10/2013. 


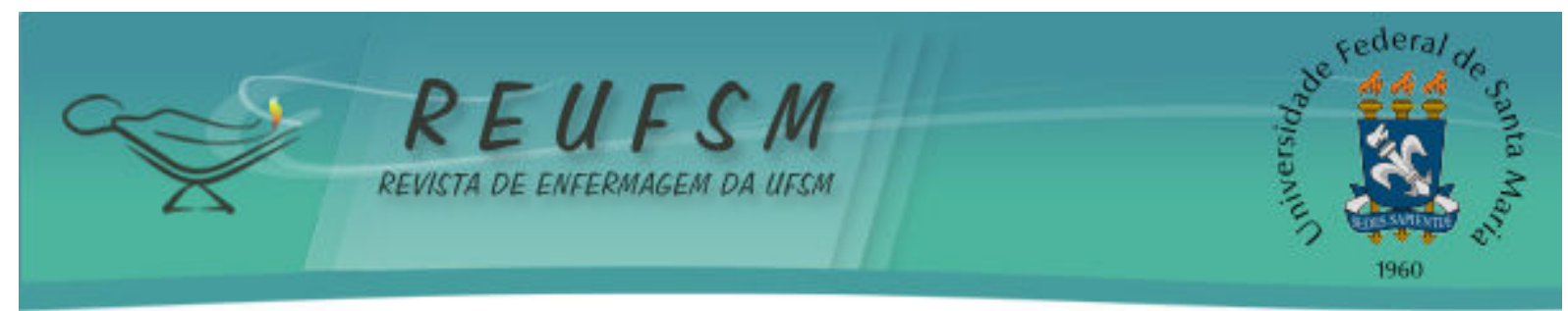

3. Gonçalez DFC, Nogueira ATO, Puggina ACG. O uso da música na assistência de enfermagem no Brasil: uma revisão bibliográfica. Cogitare Enferm, 2008, out-dez; 13(4):591-6.

4. Bradt J, Dileo C, Grocke D. Music interventions for mechanically ventilated patients. Cochrane Database of Systematic Reviews. In: The Cochrane Library, 2010.

5. Sarkamo T, Tervaniemi M, Laitinen L, Forsblom A, Soinila S, Mikkonen M, et al. Music listening enhances cognitive recovery and mood after middle cerebral artery stroke. Brain Advance Access.Fev, 2008.

6. Puggina ACG. O uso da música e de estímulos vocais em pacientes em estado de coma: relação entre estímulo auditivo, sinais vitais, expressão facial e Escala de Coma de Glasgow e Ramsay. [Dissertação] São Paulo: Escola de Enfermagem da Universidade de São Paulo, 2006.

7. Campos SM, Correia CMF, Muszkat M. Música e Neurociências. Rev Neurociênc, 2000; 8 (2): 70-75.

8. Dudeque N. Influências nas Bachianas Brasileiras: A Cantilena da Bachianas Brasileiras n. 5 e no "Trenzinho do Caipira". In: Simpósio Internacional Villa-Lobos - Universidade de São Paulo; 2009.p.65-74.

9. Ministério da Saúde (Brasil). Diretrizes de atenção à pessoa com traumatismo crânioencefálico de 03 de Janeiro de 2013. Brasília: Ministério da Saúde. Disponível em: <http://portalsaude.saude.gov.br/portalsaude> Acesso em 06/12/2013.

10. R Development Core Team, 2011. R: A language and environment for statistical computing. R Foundation for Statistical Computing, Vienna, Austria. ISBN 3-900051-07-0, URL <http://www.R-project.org/>. Acesso em 10/09/2013.

11. Conselho Nacional de Saúde. Resolução n. 466 de 12 de dezembro de 2012. Aprova as diretrizes e normas regulamentadoras de pesquisa envolvendo seres humanos. Disponível em: <http: //conselho.saude.gov.br/resolucoes/2012/Res0466.pdf> Acesso em 06/12/2013.

12. Puggina ACG. Análise das respostas vitais, faciais e tônus muscular frente ao estímulo da música ou mensagem em pacientes em coma, estado vegetativo ou sedado. [Tese] São Paulo: Escola de Enfermagem da Universidade de São Paulo, 2011.

13. Lima MVC, Guimarães RMO, Silva GPF, Mont'alverne DGB. Perfil Clínico e Desmame Ventilatório de Pacientes Acometidos por Traumatismo Crânio-Encefálico. Rev Neurociênc, 2012; 20(3):354-9.

14. Carvalho CRR, Toufen Junior C, Franca SA. Ventilação mecânica: princípios, análise gráfica e modalidades ventilatórias. III Consenso Brasileiro de Ventilação Mecânica. J Bras Pneumol, 2007;33(Supl2):S54-S70.

15. Bishop, D.T., Karageorghis, C.I., \& Kinrade, N.P. (2009). Effects of Musically-Induced Emotions on Choice Reaction Time Performance [Versao electronica], The Sport Psychologist,23 (1): 59-76. In: Simões MGN. Avaliação dos efeitos combinados do exercício físico e da música na motivação para o exercício, nos estados de humor e na função cognitiva. [dissertação] Gambelas: Faculdade de Ciências Humanas e Sociais da Universidade do Algarve, 2010. 173p.

16. Staum, M.J., \& Brotons, M. (2000). The effect of music amplitude on the relaxation response [Versao electronica], J Music Ther., 37(1):22-39. In: Simões MGN. Avaliação dos efeitos combinados do exercício físico e da música na motivação para o exercício, nos 


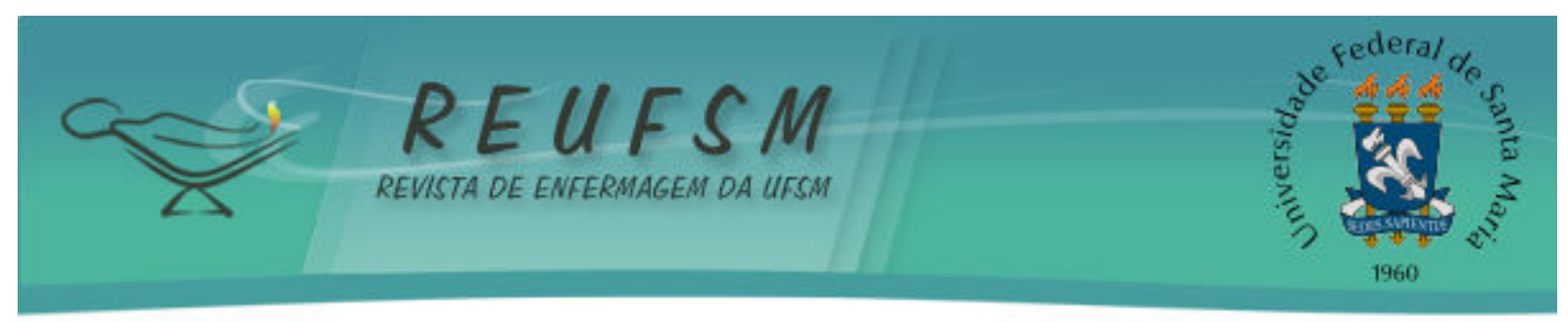

estados de humor e na função cognitiva. [dissertação] Gambelas: Faculdade de Ciências Humanas e Sociais da Universidade do Algarve, 2010. 173p.

17. Hughes, JR, Daaboul Y, Fino JJ, Shaw, GL. The "Mozart effect" on epileptiform activity. Clinical Eletrooencephalography, 29(3):109-19, 1998. In: Campos SM, Correia CMF, Muszat M. Música e Neurociências. Rev. Neurociências 8 (2): 70-75, 2000.

Data de recebimento: $14 / 03 / 2014$

Data de aceite: $24 / 11 / 2014$

Contato com autor responsável: Lara Adrianne Garcia Paiano

Endereço postal: Sala 74, bloco de salas de aula da Unioeste Campus de Cascavel. Rua Universitária 2069, Jardim Universitário. CEP 85819-110. Cascavel - PR - Brasil.

E-mail: laraagp@hotmail.com 